\title{
Discussion on seepage governing equations for low permeability reservoirs with a threshold pressure gradient
}

\author{
Guofeng $\operatorname{Han}^{1 *}$, Yuewu Liu ${ }^{1}$, Kumar Nawnit ${ }^{2}$, Yingfang Zhou ${ }^{3}$ \\ ${ }^{1}$ Institute of Mechanics, Chinese Academy of Sciences, Beijing 100190, P. R. China \\ ${ }^{2}$ State Key laboratory of Hydroscience and Engineering, Tsinghua University, Beijing 100084, P. R. China \\ ${ }^{3}$ School of Engineering, University of Aberdeen, Aberdeen AB243UE, United Kingdom \\ (Received May 18, 2018; revised June 8, 2018; accepted June 9, 2018; available online June 14, 2018)
}

\section{Citation:}

Han, G., Liu, Y., Nawnit, K., Zhou, Y. Discussion on seepage governing equations for low permeability reservoirs with a threshold pressure gradient. Advances in Geo-Energy Research, 2018, 2(3): 245-259, doi:

10.26804/ager.2018.03.03.

\section{Corresponding author:}

*E-mail: hgf_xg@126.com

\section{Keywords:}

Low permeability

flow in porous media

governing equation

non-Darcy

threshold pressure gradient

\begin{abstract}
:
Low permeability reservoirs account for an increasing proportion of oil production. Threshold pressure gradient is an important factor that governs the flow in low permeability porous media. The 1-D seepage governing equation (SGE) for low permeability porous media can be derived from the 1-D core flooding experimental rule. In the literature, for isotropic porous media, the SGE with a threshold pressure gradient (TPG) in Cartesian and cylinder coordinate systems are incompatible to each other. In addition, irrational results were found in simulation using SGEs in the Cartesian coordinate system.

In this study, 3-D SGEs with a TPG in the Cartesian coordinate system and for radial flow in the cylindrical coordinate system are derived from the vector form of the seepage velocity in 3-D domain which is transformed from the 1-D seepage velocity vector. The 1D equation degenerated from the 3-D SGE of low permeability media is in accordance with the 1-D SGE. The derived SGE of low permeability porous media in Cartesian coordinate systems is consistent with that in cylindrical coordinate systems. So, the contradiction of SGEs with a TPG in literature is resolved.

For anisotropic reservoirs with a TPG, with the assumption that the impeding of a TPG to flow in porous media occurs in the opposite direction of the seepage velocity vector, the general seepage initiation condition for anisotropic porous media with a TPG is derived. The SGEs for anisotropic porous media with a TPG under a specific condition in Cartesian coordinate systems and for radial flow in cylindrical coordination the systems are derived, and then are degenerated to isotropic cases. It is found that a simple form of the SGE anisotropic porous media with a TPG can only be derived when the flow is radial. So, it is suggested that numerical simulations for anisotropic porous media with a TPG should use the equation set composed by the pressure and seepage velocity vector. The analysis also indicates that a TPG of anisotropic reservoirs is a two-order tensor, and cannot be represented by a vector. However, the current form of effective pressure gradient requires further investigation.
\end{abstract}

\section{Introduction}

Along with high quality oil/gas reserves' gradual exhaustion, low-permeability reservoirs contribute to a large proportion of current petroleum production. However, amount of experimental studies have shown that low-permeability reservoirs, unlike conventional reservoirs, have non-linear permeability characteristics between flow rate and pressure gradient and there is a threshold pressure gradient (TPG) to archive effective seepage (Thomas et al., 1968; Prada and Civan, 1999; Zeng et al., 2010; Zeng et al., 2012). The existence of a TPG makes the flow of liquid through low permeability reservoirs have many special properties. Based on a number of experimental studies on low permeability cores ( $\mathrm{Li}$ et al., 2008; Xiong, 2009; Ding et al., 2014), a variety of constitutive relationships between pressure gradients and seepage rates have been proposed (Dudgeon, 1966; Prada and Civan, 1999; Li et al., 2016; Wang and Sheng, 2017). Combining with the specific conditions of oil and gas reservoirs, various governing equations with a TPG were established. These governing equations are widely used in reservoir engineering, including well testing analysis (Sun et al., 2010; Guo et al., 2012; Zhao et al., 2013, 2015a, 2015b; Escobar et al., 2015; Diwu et al., 2018), productivity evaluation (Zhu et al., 2011; Wu et al., 2014; Song et al., 2015; Tian et al., 2018), and rate transient 
analysis (Zeng et al., 2016, 2018). Due to the presence of a TPG, the boundary of flow in low permeability reservoirs changes over time, i.e., the moving boundary (Liu et al., 2012, 2016; Wang et al., 2015). Within the moving boundary, oil and gas could flow. So, there exists an initiation condition for seepage. At present, the governing equations used in the studies were mainly for one-dimensional linear flow and radial flow. Although 2-dimensional or 3-dimensional governing equations with TPGs are used in reservoir simulations (Xu et al., 2012; Civan, 2013, 2017; Liu and Wu, 2015; Liu, 2017), their correctness has not been validated.

It is observed that the general seepage governing equation (SGE) with a TPG in Cartesian coordinate systems reported in the literature cannot be transformed into its form in cylindrical coordinate systems for radial flow (see Section 5.1 for details). Chen (2011) claimed that the TPG of linear flow cannot be used for planar radial flow. As Bingham fluids have a TPG, Liu (2013) used the commercial simulation software CMG's Bingham module to simulate well productions in an isotropic homogeneous reservoir with a TPG, and found that the pressure distribution is not radially symmetric which does not conform to physical laws. Therefore, for the SGE with a TPG in 2-dimensional and 3-dimensional cases, it is not completely clear, and it needs further investigations.

At present, researches on flow in low-permeability reservoirs with a TPG is primarily focused on isotropic reservoirs. However, the actual reservoir permeability may be anisotropic, and it is essential to study the SEG for anisotropic lowpermeability reservoirs with a TPG.

Based on the representation of a TPG in 3-dimensional domain, the SGEs for isotropic and anisotropic reservoirs in Cartesian and cylindrical coordinate systems are derived. Then, the consistency of SEGs for isotropic reservoirs, the form of the SEGs and the representation of a TPG are discussed. At last, the SEGs of the anisotropic and isotropic reservoirs are compared.

\section{Flow in low-permeability porous media}

A large number of experiments have shown that the relation between a flow velocity and a pressure gradient in low permeability cores does not follow Darcy's law, namely it is not a straight line through the origin of coordinates (Fig. 1). This figure illustrates that the flow velocity vs pressure gradient curves' $x$-axis (Pressure Gradient) intersection not equals to zero, and the corresponding pressure gradient is named as TPG (see point A). It is also shown that the relationship between seepage velocities and pressure gradients is not linear at small pressure gradient. It becomes linear when the pressure gradient increases, while the extension of the straight line does not pass through the origin, and the intersection point $\mathrm{B}$ is the pseudo-threshold pressure gradient (PTPG). A distinction between a TPG and a PTPG will not be made in the following sections. There are many equations for describing low-permeability seepage laws, including power law functions, exponential functions, linear functions with a single parameter, linear functions with multi-parameters, etc (Diwu et al., 2018). For one-dimensional core flooding exper-

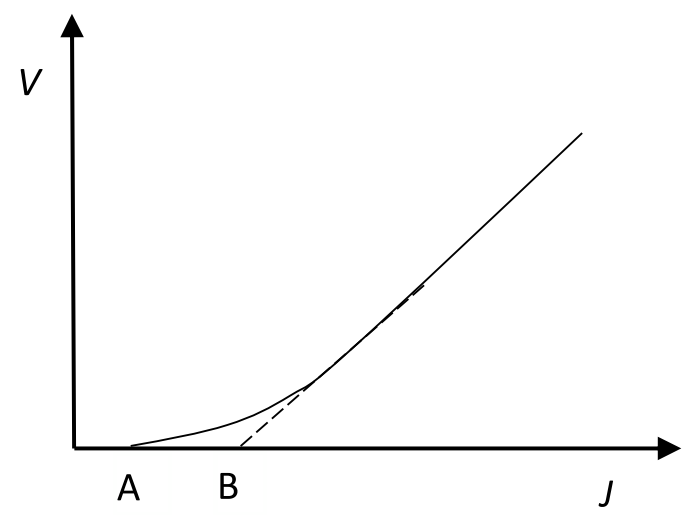

Fig. 1. Schematic plot of non-Darcy flow for low-permeability cores.

iments, the following form of linear equation is normally used to describe the relationship between flow rates and pressure gradients (Prada et al., 1999):

$$
V= \begin{cases}-\frac{K}{\mu} J\left(1-\frac{\lambda}{|J|}\right) & |J|>\lambda \\ 0 & |J|<\lambda\end{cases}
$$

It should be noted that the bold symbols $\mathbf{V}, \mathbf{J}, \mathbf{K}$, and $\boldsymbol{\lambda}$ represent the velocity vector, pressure gradient vector, permeability tensor, and TPG tensor in 3-dimensional domain, respectively, and the un-bold symbols indicate their 1dimensional domain Scalar.

The direction of a flow velocity is not necessarily consistent with the coordinate axis in 3-dimensional domain, and thus it is worth to revisit the SGE. For convenience, the influence of gravity is neglected in Eq. (1). If including gravity affect and assuming it acts downward along the $z$-coordinate, the pressure gradient in $z$-coordinate, $\partial p / \partial z$, should be replaced with $\partial p / \partial z+\rho g$. It has to be noted that the onedimensional form of seepage Eq. (1) is based on the direction consistency of the seepage velocity vector, the pressure gradient vector, and the spatial axis. When the direction of a seepage velocity vector is not parallel to any axis, the seepage velocity vector and the pressure gradient vector can be written in a 3dimensional form. For isotropic reservoirs, the direction of a seepage velocity vector is consistent with that of the pressure gradient vector. When the pressure gradient is greater than the TPG, the velocity vector in 3-dimensional coordinates can be obtained from Eq. (1) (Dou et al., 2014):

$$
\begin{aligned}
\boldsymbol{V} & =\left[\begin{array}{l}
V_{x} \\
V_{y} \\
V_{z}
\end{array}\right]=-\frac{K}{\mu} \boldsymbol{J}\left(1-\frac{\lambda}{|\boldsymbol{J}|}\right) \\
& =-\frac{K}{\mu}\left(\begin{array}{l}
\frac{\partial p}{\partial x} \\
\frac{\partial p}{\partial y} \\
\frac{\partial p}{\partial z}
\end{array}\right)\left(1-\frac{\lambda}{\sqrt{\left(\frac{\partial p}{\partial x}\right)^{2}+\left(\frac{\partial p}{\partial y}\right)^{2}+\left(\frac{\partial p}{\partial z}\right)^{2}}}\right)
\end{aligned}
$$


This equation is only a result of the coordinate transformation of the seepage velocity vector Eq. (1) . The permeability of anisotropic reservoirs is a second-order tensor, and the general form in the Cartesian coordinate system $(x, y, z)$ is (Kong, 2010):

$$
\boldsymbol{K}=\left[\begin{array}{lll}
K_{x x} & K_{x y} & K_{x z} \\
K_{y x} & K_{y y} & K_{y z} \\
K_{z x} & K_{z y} & K_{z z}
\end{array}\right]
$$

For ease of analysis, assuming the axes coincides with the three principal axes of the tensor, respectively, then, Eq. (3) becomes (Kong, 2010):

$$
\boldsymbol{K}=\left[\begin{array}{ccc}
K_{x x} & 0 & 0 \\
0 & K_{y y} & 0 \\
0 & 0 & K_{z z}
\end{array}\right]
$$

When $K_{x x}=K_{y y}=K_{z z}$, it is the permeability tensor for isotropic reservoirs. For permeability of anisotropic reservoirs, the TPG can also be represented by a second-order tensor (discussed in detail in Section 5.4). Also assuming its major axes are consistent with these of the permeability tensor, for simplicity, the following Eq. (5) uses the linear form of the TPG.

$$
\boldsymbol{\lambda}=\left[\begin{array}{ccc}
\lambda_{x} & 0 & 0 \\
0 & \lambda_{y} & 0 \\
0 & 0 & \lambda_{z}
\end{array}\right]
$$

For isotropic reservoirs, $\lambda_{x}=\lambda_{y}=\lambda_{z}=\lambda$. In onedimensional low-permeability core experiments, the impeding effect of a TPG on the seepage flow points in its negative direction. In the 3-dimensional domain, it is assumed that the impeding effect of the anisotropic reservoir TPG tensor is similar as in the $1 \mathrm{D}$ case. So the effective pressure gradient for seepage in the 3-dimensional domain is:

$$
\begin{aligned}
\boldsymbol{J}_{e} & =\boldsymbol{J}-\left(-\boldsymbol{\lambda} \cdot \frac{\boldsymbol{V}}{|\boldsymbol{V}|}\right) \\
& =\boldsymbol{J}+\boldsymbol{\lambda} \cdot \frac{\boldsymbol{V}}{|\boldsymbol{V}|}
\end{aligned}
$$

Therefore, the 3-dimensional domain seepage velocity is:

$$
\begin{aligned}
& \boldsymbol{V}=-\frac{\boldsymbol{K}}{\mu} \cdot\left(\boldsymbol{J}+\boldsymbol{\lambda} \cdot \frac{\boldsymbol{V}}{|\boldsymbol{V}|}\right) \\
& =-\frac{1}{\mu}\left\{\left(\begin{array}{c}
K_{x} \frac{\partial p}{\partial x} \\
K_{y} \frac{\partial p}{\partial y} \\
K_{z} \frac{\partial p}{\partial z}
\end{array}\right)+\left[\begin{array}{c}
\lambda_{x} K_{x} V_{x} \\
\lambda_{y} K_{y} V_{y} \\
\lambda_{z} K_{z} V_{z}
\end{array}\right] \frac{1}{\sqrt{V_{x}^{2}+V_{y}^{2}+V_{z}^{2}}}\right\}
\end{aligned}
$$

This equation could be reformulated as:

$$
\left[\begin{array}{l}
\left(\mu+\frac{\lambda_{x} K_{x}}{\sqrt{V_{x}^{2}+V_{y}^{2}+V_{z}^{2}}}\right) V_{x} \\
\left(\mu+\frac{\lambda_{y} K_{y}}{\sqrt{V_{x}^{2}+V_{y}^{2}+V_{z}^{2}}}\right) V_{y} \\
\left(\mu+\frac{\lambda_{z} K_{z}}{\sqrt{V_{x}^{2}+V_{y}^{2}+V_{z}^{2}}}\right) V_{z}
\end{array}\right]=-\left(\begin{array}{l}
K_{x} \frac{\partial p}{\partial x} \\
K_{y} \frac{\partial p}{\partial y} \\
K_{z} \frac{\partial p}{\partial z}
\end{array}\right)
$$

Eq. (8) is then written as:

$$
\begin{gathered}
1=\frac{\left(K_{x} \frac{\partial p}{\partial x}\right)^{2}}{\left(\mu \sqrt{V_{x}^{2}+V_{y}^{2}+V_{z}^{2}}+\lambda_{x} K_{x}\right)^{2}} \\
+\frac{\left(K_{y} \frac{\partial p}{\partial y}\right)^{2}}{\left(\mu \sqrt{V_{x}^{2}+V_{y}^{2}+V_{z}^{2}}+\lambda_{y} K_{y}\right)^{2}} \\
+\frac{\left(K_{z} \frac{\partial p}{\partial z}\right)^{2}}{\left(\mu \sqrt{V_{x}^{2}+V_{y}^{2}+V_{z}^{2}}+\lambda_{z} K_{z}\right)^{2}}
\end{gathered}
$$

The seepage flow occurs only when the pressure gradient is greater than the TPG. It implies $\sqrt{V_{x}^{2}+V_{y}^{2}+V_{z}^{2}}>0$. So, the following can be written as:

$$
\left(\frac{1}{\lambda_{x}} \frac{\partial p}{\partial x}\right)^{2}+\left(\frac{1}{\lambda_{y}} \frac{\partial p}{\partial y}\right)^{2}+\left(\frac{1}{\lambda_{z}} \frac{\partial p}{\partial z}\right)^{2}>1
$$

Eq. (10) is the general form of seepage initiation condition for an anisotropic reservoir with a TPG. Eq. (10) could be simplified as the same seepage initiation as shown in Eq. (1) for isotropic reservoirs.

\section{Governing equations with a TPG for isotropic reservoirs}

In the Cartesian coordinate system $(x, y, z)$, the $z$-axis is the direction of gravity. Then the seepage flow rate (2) is written in another form as:

$$
\begin{aligned}
& \left\{\begin{array}{l}
V_{x}=-\frac{K}{\mu}\left(\frac{\partial p}{\partial x}-\lambda_{\lambda x}\right) \\
V_{y}=-\frac{K}{\mu}\left(\frac{\partial p}{\partial z}-\lambda_{\lambda y}\right) \\
V_{z}=-\frac{K}{\mu}\left(\frac{\partial p}{\partial z}-\lambda_{\lambda z}\right)
\end{array}\right. \\
& \sqrt{\left(\frac{\partial p}{\partial x}\right)^{2}+\left(\frac{\partial p}{\partial y}\right)^{2}+\left(\frac{\partial p}{\partial z}\right)^{2}}>\lambda
\end{aligned}
$$


where

$$
\left\{\begin{array}{l}
\lambda_{\lambda x}=\frac{\lambda_{x} K_{x} \frac{\partial p}{\partial x}}{\sqrt{\left(K_{x} \frac{\partial p}{\partial x}\right)^{2}+\left(K_{y} \frac{\partial p}{\partial y}\right)^{2}+\left(K_{z} \frac{\partial p}{\partial z}\right)^{2}}} \\
\lambda_{\lambda y}=\frac{\lambda_{y} K_{y} \frac{\partial p}{\partial y}}{\sqrt{\left(K_{x} \frac{\partial p}{\partial x}\right)^{2}+\left(K_{y} \frac{\partial p}{\partial y}\right)^{2}+\left(K_{z} \frac{\partial p}{\partial z}\right)^{2}}} \\
\lambda_{\lambda_{z}}=\frac{\lambda_{z} K_{z} \frac{\partial p}{\partial z}}{\sqrt{\left(K_{x} \frac{\partial p}{\partial x}\right)^{2}+\left(K_{y} \frac{\partial p}{\partial y}\right)^{2}+\left(K_{z} \frac{\partial p}{\partial z}\right)^{2}}}
\end{array}\right.
$$

Note that for permeability of isotropic reservoirs, $K_{x x}=K_{y y}$ $=K_{z z}$ and $\lambda_{x}=\lambda_{y}=\lambda_{z}=\lambda$ in the above equation. It should be noted that $\lambda_{\lambda x}, \lambda_{\lambda y}$ and $\lambda_{\lambda z}$ are not the components of the TPG in the $x, y$, and $z$ directions, respectively, but are simply the symbols introduced for writing concisely.

Here, the fluid compressibility $\left(c_{f}\right)$ and the pore compressibility $\left(c_{r}\right)$ are defined as:

$$
\begin{gathered}
c_{f}=\frac{1}{\rho} \frac{d \rho}{d p} \\
c_{r}=\frac{1}{\phi} \frac{d \phi}{d p}
\end{gathered}
$$

And the total compressibility can be defined as:

$$
c_{t}=c_{r}+c_{f}
$$

Substituting velocity Eq. (11) into the continuity Eq. (16):

$$
\begin{gathered}
\frac{\partial(\phi \rho)}{\partial t}+\nabla \cdot(\rho \boldsymbol{V})=\rho q \\
(16) \quad \begin{array}{l}
\text { a second order continuous derivative with respect } \\
z \text { for the pressure, the governing equation is: }
\end{array} \\
\left(\frac{\partial^{2} p}{\partial x^{2}}+\frac{\partial^{2} p}{\partial y^{2}}+\frac{\partial^{2} p}{\partial z^{2}}\right)\left(1-\frac{\lambda}{\sqrt{\left(\frac{\partial p}{\partial x}\right)^{2}+\left(\frac{\partial p}{\partial y}\right)^{2}+\left(\frac{\partial p}{\partial z}\right)^{2}}}\right)^{\lambda} \\
+\frac{\left(\frac{\partial p}{\partial x}\right)^{2} \frac{\partial^{2} p}{\partial x^{2}}+\left(\frac{\partial p}{\partial y}\right)^{2} \frac{\partial^{2} p}{\partial y^{2}}+\left(\frac{\partial p}{\partial z}\right)^{2} \frac{\partial^{2} p}{\partial z^{2}}}{\left[\left(\frac{\partial p}{\partial x}\right)^{2}+\left(\frac{\partial p}{\partial y}\right)^{2}+\left(\frac{\partial p}{\partial z}\right)^{2}\right]^{\frac{3}{2}}}\left[\begin{array}{l}
\left.+2 \frac{\partial p}{\partial x} \frac{\partial p}{\partial y} \frac{\partial^{2} p}{\partial x \partial y}+2 \frac{\partial p}{\partial x} \frac{\partial p}{\partial z} \frac{\partial^{2} p}{\partial x \partial z}+2 \frac{\partial p}{\partial y} \frac{\partial p}{\partial z} \frac{\partial^{2} p}{\partial y \partial z}\right]=\frac{\phi \mu c_{t}}{K} \frac{\partial p}{\partial t} \\
\sqrt{\left(\frac{\partial p}{\partial x}\right)^{2}+\left(\frac{\partial p}{\partial y}\right)^{2}+\left(\frac{\partial p}{\partial z}\right)^{2}}>\lambda
\end{array}\right.
\end{gathered}
$$

It yields Eq. (17) in the $x$ direction:

$$
\begin{aligned}
\frac{\partial\left(\rho V_{x}\right)}{\partial x} & =-\frac{K}{\mu} \frac{\partial}{\partial x}\left(\rho \frac{\partial p}{\partial x}-\rho \lambda_{\lambda x}\right) \\
& =-\frac{K \rho}{\mu}\left[c_{f}\left(\frac{\partial p}{\partial x}\right)^{2}+\frac{\partial^{2} p}{\partial x^{2}}-\frac{\partial \lambda_{\lambda x}}{\partial x}-c_{f} \lambda_{\lambda x} \frac{\partial p}{\partial x}\right]
\end{aligned}
$$

When deriving the SGEs, it is generally assumed that the quadratic term of the pressure gradient can be ignored. This hypothesis is also adopted here. Substituting Eq. (12) into Eq. (17), it yields:

$$
\begin{aligned}
& \frac{\partial\left(\rho V_{x}\right)}{\partial x}= \\
& -\frac{\rho K}{\mu}\left\{\begin{array}{c}
\frac{\partial^{2} p}{\partial x^{2}}\left(1-\frac{\lambda}{\sqrt{\left(\frac{\partial p}{\partial x}\right)^{2}+\left(\frac{\partial p}{\partial y}\right)^{2}+\left(\frac{\partial p}{\partial z}\right)^{2}}}\right) \\
+\frac{\partial p}{\partial x} \frac{\lambda}{\left[\left(\frac{\partial p}{\partial x}\right)^{2}+\left(\frac{\partial p}{\partial y}\right)^{2}+\left(\frac{\partial p}{\partial z}\right)^{2}\right]^{\frac{3}{2}} \times} \\
\left(\frac{\partial p}{\partial x} \frac{\partial^{2} p}{\partial x^{2}}+\frac{\partial p}{\partial y} \frac{\partial^{2} p}{\partial x \partial y}+\frac{\partial p}{\partial z} \frac{\partial^{2} p}{\partial x \partial z}\right)
\end{array}\right\}
\end{aligned}
$$

Similar results can be derived for the $y$ and $z$ directions, which are not explicitly written here. Also, the first item of the left side of the continuity Eq. (16) can be transformed as:

$$
\frac{\partial(\rho \phi)}{\partial t}=\rho \frac{d \phi}{d p} \frac{\partial p}{\partial t}+\phi \frac{d \rho}{d p} \frac{\partial p}{\partial t}=\rho \phi c_{r} \frac{\partial p}{\partial t}+\rho \phi c_{f} \frac{\partial p}{\partial t}=\phi \rho c_{t} \frac{\partial p}{\partial t}
$$

Assuming that the source or sink term $q$ is 0 and there is a second order continuous derivative with respect to $x, y$, and
Eq. (20) is the 3-dimensional SGE for permeability isotropic reservoirs with a TPG in Cartesian coordinate sys- tems. Their form for radial flow in cylindrical coordinate systems is shown in Appendix 1. 


\section{Governing equations with a TPG for anisotropic reservoirs}

\subsection{Cartesian coordinate system}

It is easy to find out from Eq. (8) that the solution of the general form of seepage velocity components requires solving a unary six order equation. Obtaining the analytical solution of this equation is difficult. To obtain a simple SGE, an assumption is made here. The assumption is that the direction of the seepage velocity with a TPG is the same as that without a TPG, i.e.,

$$
\frac{\boldsymbol{V}}{|\boldsymbol{V}|}=-\frac{\boldsymbol{K} \boldsymbol{J}}{|\boldsymbol{K} \boldsymbol{J}|}
$$

With the assuption Eq. (21), the SGE and seepage initiation condition for anisotropic reservoirs with a TPG in Cartesian coordinate systems can be obtained. The specific derivation and results can be found in Appendix 2.1. At the same time, it can be found that this equation requires $K_{x} \lambda_{x}=K_{y} \lambda_{y}=$ $K_{z} \lambda_{z}$.

\subsection{Cylindrical coordinate system}

In the cylindrical coordinate system $(r, \theta, z)$, the relationship between its base vector and that of the Cartesian coordinate system is represented as:

$$
\left\{\begin{array}{l}
\mathbf{e}_{r}=\cos \theta \overline{\mathbf{e}}_{x}+\sin \theta \overline{\mathbf{e}}_{y} \\
\mathbf{e}_{\theta}=-\sin \theta \overline{\mathbf{e}}_{x}+\cos \theta \overline{\mathbf{e}}_{\mathbf{y}} \\
\mathbf{e}_{z}=\overline{\mathbf{e}}_{z}
\end{array}\right.
$$

According to the theory of tensor analysis (Huang et al., 2003), the permeability tensor in the cylindrical coordinate system can be obtained from the permeability tensor Eq. (4) in the Cartesian coordinate system using Eq. (22) as:

$$
\left[\begin{array}{ccc}
K_{x} \cos ^{2} \theta+K_{y} \sin ^{2} \theta & \left(K_{y}-K_{x}\right) \cos \theta \sin \theta & 0 \\
\left(K_{y}-K_{x}\right) \cos \theta \sin \theta & K_{x} \sin ^{2} \theta+K_{y} \cos ^{2} \theta & 0 \\
0 & 0 & K_{z}
\end{array}\right]
$$

Similarly, the TPG tensor in the cylindrical coordinate system can be obtained from the TPG tensor Eq. (5) in the Cartesian coordinate system as follow:

$$
\left[\begin{array}{ccc}
\lambda_{x} \cos ^{2} \theta+\lambda_{y} \sin ^{2} \theta & \left(\lambda_{y}-\lambda_{x}\right) \cos \theta \sin \theta & 0 \\
\left(\lambda_{y}-\lambda_{x}\right) \cos \theta \sin \theta & \lambda_{x} \sin ^{2} \theta+\lambda_{y} \cos ^{2} \theta & 0 \\
0 & 0 & \lambda_{z}
\end{array}\right]
$$

While considering the general SGE, the calculation will become complicated. SEGs are much simpler for the axissymmetrical condition, but the equations are also very complicated because of the seepage velocity in the $z$-direction. The radial flow governing equation is commonly used in well testing. Here, only the radial flow governing equation is derived. The results of the derivation are shown in Appendix 2.2. This governing equation requires $K_{x} \lambda_{x}=K_{y} \lambda_{y}$.

\section{Discussion}

\subsection{The consistency of governing equations in differ- ent coordinate systems}

In the case of a 1-dimensional flow, the governing Eq. (20) in the Cartesian coordinate systems can be simplified into:

$$
\frac{\partial^{2} p}{\partial x^{2}}=\frac{\phi \mu c_{t}}{K} \frac{\partial p}{\partial t}
$$

Eq. (25) is consistent with that in the literature (Liu et al., 2012), which demonstrates the credibility of the derivation above. Also, it can be seen that any item in the governing Eq. (20) cannot be ignored. The following equation for lowpermeability reservoirs in Cartesian coordinate systems appears in the literature (Kong, 2010; Lu, 2012, 2014):

$$
\frac{\partial^{2} p}{\partial x^{2}}+\frac{\partial^{2} p}{\partial y^{2}}+\frac{\partial^{2} p}{\partial z^{2}}=\frac{\phi \mu c_{t}}{K} \frac{\partial p}{\partial t}
$$

And governing equations in cylindrical coordinate systems (Kong, 2010; Zhao et al., 2015) is presented as:

$$
\begin{gathered}
\frac{\partial^{2} p}{\partial r^{2}}+\frac{1}{r} \frac{\partial p}{\partial r} \mp \frac{\lambda}{r}=\frac{\phi \mu c_{t}}{K} \frac{\partial p}{\partial t} \\
\left|\frac{\partial p}{\partial r}\right|>\lambda
\end{gathered}
$$

The negative sign preceding the last item on the left of the Eq. (27) corresponds to a production well, and the positive sign corresponds to an injection well.

The governing equation in the cylindrical coordinate systems for radial flow could also be derived from Eq. (26) as:

$$
\frac{\partial^{2} p}{\partial r^{2}}+\frac{1}{r} \frac{\partial p}{\partial r}=\frac{\phi \mu c_{t}}{K} \frac{\partial p}{\partial t}
$$

It is observed that Eq. (28) is inconsistent with Eq. (27), and it lacks of a term corresponding to the TPG. Thus it is questionable. Also, it can be seen that the Eq. (20) and Eq. (28) in Cartesian coordinate systems are different from each other. While the governing equations Eqs. (A1-6) and (27) in the cylindrical coordinate systems are consistent.

Eq. (20) for low-permeability reservoirs in Cartesian coordinate systems is derived from Eq. (17) by ignoring the quadratic terms of the pressure gradient, which gives Eq. (29):

$$
\frac{\partial\left(\rho V_{x}\right)}{\partial x}=-\frac{K \rho}{\mu}\left[\frac{\partial^{2} p}{\partial x^{2}}-\frac{\partial \lambda_{\lambda x}}{\partial x}-c_{f} \lambda_{\lambda x} \frac{\partial p}{\partial x}\right]
$$

While considering radial flow:

$$
\lambda_{\lambda x}=\frac{\lambda}{\left|\frac{\partial p}{\partial r}\right|} \frac{\partial p}{\partial r} \cos \theta=\frac{\lambda}{\left|\frac{\partial p}{\partial r}\right|} \frac{\partial p}{\partial r} \frac{x}{r}
$$

and,

$$
\frac{\partial \lambda_{\lambda x}}{\partial x}=\frac{\lambda}{\left|\frac{\partial p}{\partial r}\right|} \frac{\partial p}{\partial r} \frac{\partial}{\partial x}\left(\frac{x}{r}\right)=\frac{\lambda}{\left|\frac{\partial p}{\partial r}\right|} \frac{\partial p}{\partial r} \frac{y^{2}}{r^{3}}
$$


and it results in:

$$
\begin{aligned}
& \lambda_{\lambda x} \frac{\partial p}{\partial x}+\lambda_{\lambda y} \frac{\partial p}{\partial y}=\frac{\lambda}{\left|\frac{\partial p}{\partial r}\right|}\left(\frac{\partial p}{\partial r} \frac{x}{r} \frac{\partial p}{\partial x}+\frac{\partial p}{\partial r} \frac{y}{r} \frac{\partial p}{\partial y}\right) \\
& =\frac{\lambda}{\left|\frac{\partial p}{\partial r}\right|} \frac{\partial p}{\partial r}\left(\frac{\partial p}{\partial x} \cos \theta+\frac{\partial p}{\partial y} \sin \theta\right)=\frac{\lambda}{\left|\frac{\partial p}{\partial r}\right|}\left(\frac{\partial p}{\partial r}\right)^{2}=\lambda\left|\frac{\partial p}{\partial r}\right|
\end{aligned}
$$

Substituting Eqs. (30) and (32) into Eq. (29) and taking into account the similarity of equations in the $y$-direction and $x$-direction and ignoring the quadratic terms, will result in Eqs. (A1-6). Therefore, unlike Eq. (26), the SGE Eq. (20) for low permeability reservoirs in Cartesian coordinate systems is consistent with that in cylindrical coordinate systems.

Derivation of the above Eq. (26) in the literature (Kong, 2010) is based on the seepage velocity in each direction as follows:

$$
\begin{aligned}
& \left\{\begin{array}{l}
V_{x}=-\frac{K}{\mu} \frac{\partial p}{\partial x}\left(1-\frac{\lambda_{x}}{\left|\frac{\partial p}{\partial x}\right|}\right),\left|\frac{\partial p}{\partial x}\right|>\lambda_{x} \\
V_{y}=-\frac{K}{\mu} \frac{\partial p}{\partial y}\left(1-\frac{\lambda_{y}}{\left|\frac{\partial p}{\partial y}\right|}\right),\left|\frac{\partial p}{\partial y}\right|>\lambda_{y} \\
V_{z}=-\frac{K}{\mu} \frac{\partial p}{\partial z}\left(1-\frac{\lambda_{z}}{\left|\frac{\partial p}{\partial z}\right|}\right),\left|\frac{\partial p}{\partial z}\right|>\lambda_{z}
\end{array}\right. \\
& \left\{\begin{array}{l}
\frac{\partial p}{\partial r}\left(K_{y}-K_{x}\right) \cos \theta \sin \theta+\frac{1}{r} \frac{\partial p}{\partial \theta}\left(K_{x} \sin ^{2} \theta+K_{y} \cos ^{2} \theta\right)+\frac{V_{r}}{\left|V_{r}\right|}\left(K_{y} \lambda_{y}-K_{x} \lambda_{x}\right) \cos \theta \sin \theta=0 \\
V_{r}=-\frac{1}{\mu}\left\{\begin{array}{l}
\frac{\partial p}{\partial r}\left[\frac{K_{x} \cos ^{2} \theta+K_{y} \sin ^{2} \theta}{K_{x} \lambda_{x} \cos ^{2} \theta+K_{y} \lambda_{y} \sin ^{2} \theta}-\frac{K_{y}-K_{x}}{K_{y} \lambda_{y}-K_{x} \lambda_{x}}\right] \\
+\frac{\partial p}{r \partial \theta}\left[\frac{\left(K_{y}-K_{x}\right) \cos \theta \sin \theta}{K_{x} \lambda_{x} \cos ^{2} \theta+K_{y} \lambda_{y} \sin ^{2} \theta}-\frac{K_{x} \sin ^{2} \theta+K_{y} \cos ^{2} \theta}{\left(K_{y} \lambda_{y}-K_{x} \lambda_{x}\right) \cos \theta \sin \theta}\right]
\end{array}\right\}\left(K_{x} \lambda_{x} \cos ^{2} \theta+K_{y} \lambda_{y} \sin ^{2} \theta\right) \\
\frac{\partial p}{\partial z}=0
\end{array}\right.
\end{aligned}
$$

\subsection{The form simplicity of governing equations}

For ease of writing, define:

$$
\left\{\begin{aligned}
A & =\left(K_{x} \lambda_{x} \cos ^{2} \theta+K_{y} \lambda_{y} \sin ^{2} \theta\right)\left(\frac{K_{x} \cos ^{2} \theta+K_{y} \sin ^{2} \theta}{K_{x} \lambda_{x} \cos ^{2} \theta+K_{y} \lambda_{y} \sin ^{2} \theta}-\frac{K_{y}-K_{x}}{K_{y} \lambda_{y}-K_{x} \lambda_{x}}\right) \\
& =\frac{K_{x} K_{y}\left(\lambda_{y}-\lambda_{x}\right)}{K_{y} \lambda_{y}-K_{x} \lambda_{x}} \\
B & =\left[\frac{\left(K_{y}-K_{x}\right) \cos \theta \sin \theta}{K_{x} \lambda_{x} \cos ^{2} \theta+K_{y} \lambda_{y} \sin ^{2} \theta}-\frac{K_{x} \sin ^{2} \theta+K_{y} \cos ^{2} \theta}{\left(K_{y} \lambda_{y}-K_{x} \lambda_{x}\right) \cos \theta \sin \theta}\right]\left(K_{x} \lambda_{x} \cos ^{2} \theta+K_{y} \lambda_{y} \sin ^{2} \theta\right) \\
& =\frac{K_{x} K_{y}\left(\lambda_{x} \cos ^{2} \theta+\lambda_{y} \sin ^{2} \theta\right)}{\left(K_{x} \lambda_{x} \cos ^{2} \theta+K_{y} \lambda_{y} \sin ^{2} \theta\right)\left(K_{y} \lambda_{y}-K_{x} \lambda_{x}\right) \cos \theta \sin \theta}
\end{aligned}\right.
$$


Substituting Eq. (36) into the radial flow governing Eq. (A1-2), and ignoring the source or sink terms and quadratic pressure gradient terms, the SGE Eq. (37) can be obtained.

$$
A \frac{\partial^{2} p}{\partial r^{2}}+\frac{B}{r} \frac{\partial^{2} p}{\partial r \partial \theta}+\frac{A}{r} \frac{\partial p}{\partial r}=\phi \mu c_{t} \frac{\partial p}{\partial t}
$$

It can be seen that the governing equation is not only related to the pressure gradient in the $r$-direction but also to that in the $\theta$-direction. Unlike the governing Eq. (A2.2-6), Eq. (37) requires $K_{x} \lambda_{x} \neq K_{y} \lambda_{y}$. Combining with the governing Eq. (A2.2-6), it can be known that when it is a radial flow, a relatively simple form of a SGE with a TPG for anisotropic reservoirs can be obtained.

The isotropic low permeability SGE Eq. (20) in the Cartesian coordinate systems is very complex. Although many assumptions have been made in the derivation of equations for anisotropic low-permeability reservoirs, its form in the Cartesian coordinate systems is still complex. The general form of SGEs for an anisotropic reservoir with a TPG becomes more complicated. Therefore, in general, the SGEs for low permeability reservoirs with a TPG are not suitable for simulations and analysis. When performing numerical simulations, it is more appropriate to use equation sets including the seepage velocity and pressure as variables.

\subsection{Comparisons of anisotropic and isotropic govern- ing equations}

When $K_{x}=K_{y}=K_{z}$ and $\lambda_{x}=\lambda_{y}=\lambda_{z}$, the governing Eqs. (A2.1-6) and (A2.2-6) degenerate into the SGE for isotropic reservoirs with a TPG in the Cartesian coordinate systems and the SGE for radial flow in cylindrical coordinate systems, respectively. They are consistent with the results obtained by Eq. (20) and Eq. (A1-6), respectively. Therefore, the SGEs for an anisotropic reservoir with a TPG are justified here.

It can be verified that the condition of seepage initiation in Eq. (A2.1-6) and equation Eq. (A2.2-6) is a special case of the general initiation condition Eq. (10) for seepage with a TPG, which looks like an ellipsoidal equation. When $K_{x}=$ $K_{y}=K_{z}$ and $\lambda_{x}=\lambda_{y}=\lambda_{z}$, the seepage initiation condition for isotropic reservoirs can be obtained from Eq. (10).

For anisotropic reservoirs, when there is a TPG, it can be seen from the previous derivation that, in general, the direction of seepage velocity in anisotropic reservoirs is not only inconsistent with the direction of the pressure gradient but also inconsistent with the direction of the seepage velocity without a TPG. This nature is not the same as that of isotropic reservoirs. Only when $K_{x} \lambda_{x}=K_{y} \lambda_{y}=K_{z} \lambda_{z}$, it can be ensured that the direction of seepage velocity with a TPG is consistent with that without a TPG. For radial flow, since the velocity in the $z$-direction is 0 , and thus it only requires $K_{x} \lambda_{x}=K_{y} \lambda_{y}$.

\subsection{Representation of a TPG}

A scalar cannot represent a TPG of anisotropic reservoirs. As the name TPG contains "pressure gradient", it is easy to



Fig. 2. The directions of the pressure gradient and the TPG.

misunderstand it as a vector. While it works just similar to a vector, and thus previously a vector is used to express a TPG (Kong, 2010). However, this representation would bring inadequacy. In Section 5.1, the contradiction between the SGE with a TPG in Cartesian coordinate systems and cylindrical coordinate systems is discussed. This could be explained by considering a TPG as a vector.

Assuming that a TPG is a vector and thus it has a direction. A TPG is often written into three component quantities $\left(\lambda_{x}, \lambda_{y}\right.$, $\left.\lambda_{z}\right)$, which can be transformed into $\left(\lambda_{x}, 0,0\right)$ by a coordinate transformation (Kong, 2010). Therefore, a coordinate system may be established as shown in Fig. 2 so that the positive $x$-axis direction coincides with the direction AB of the TPG vector $\lambda$. When the reservoir pressure gradient is along $\mathrm{PT}_{1}$ ( $\mathrm{PT}_{1}$ and $\mathrm{AB}$ are in the same direction), it should meet the following condition:

$$
\left|\frac{\partial p}{\partial x}-\lambda_{x}\right|<\left|\frac{\partial p}{\partial x}\right|
$$

Eq. (38) shows that the TPG acts as a hindrance, which is consistent with the general understanding. When the reservoir pressure gradient is along $\mathrm{PT}_{2}\left(\mathrm{PT}_{2}\right.$ and $\mathrm{AB}$ are in the opposite direction), the following holds:

$$
\left|\frac{\partial p}{\partial x}-\lambda_{x}\right|>\left|\frac{\partial p}{\partial x}\right|
$$

Eq. (39) shows that the TPG promotes the seepage. It means that when there is no pressure gradient, the fluid will also flow in the direction $\mathrm{AB}$. When the reservoir pressure gradient is along $\mathrm{PT}_{3}$ or $\mathrm{PT}_{4}$ (parallel to the $y$-axis), the following holds:

$$
\left|\frac{\partial p}{\partial y}-\lambda_{y}\right|=\left|\frac{\partial p}{\partial y}\right|
$$

Eq. (40) shows that the TPG does not work. It is unreasonable. Therefore, it is not suitable to express a TPG with a vector.

To avoid this contradiction, in the literature, the absolute value of pressure gradients is used when the governing equation is written in the component form, as described in Section 5.1, but it could still not avoid the contradiction between the 
governing equations in Cartesian and cylindrical coordinate systems. The reason for this problem is that the direction of a TPG is fixed by the vector representation (using the absolute value, it is reasonable when the pressure gradient is along $\mathrm{PT}_{1}$ and $\mathrm{PT}_{2}$, but the above situation still occurs along $\mathrm{PT}_{3}$ and $\mathrm{PT}_{4}$ ). The effect of a TPG is a vector that changes with the direction of the seepage velocity or pressure gradient. So the key is to determine how this vector changes with the seepage velocity or pressure gradient.

Additionally, the SGEs of anisotropic reservoirs must be able to degenerate into that for isotropic reservoirs. It requires that a TPG under anisotropic conditions can degenerate into that under isotropic conditions. This cannot be achieved except for a zero vector. If the TPG is a zero vector, that means there is no TPG.

A scalar and vectors are 0th order and first order tensors, respectively. They cannot represent a TPG of anisotropic reservoirs. If a second-order tensor can represent a TPG of anisotropic reservoirs, it is perfect from the point of mathematical simplicity view. When a TPG and permeability are an isotropic second-order tensor, the SGEs for anisotropic reservoirs with a TPG can be degenerated into that for isotropic reservoirs. This can be easily verified from Eq. (A2.1-6) and Eq. (A2.2-6).

As discussed above, it is assumed that a TPG hinders the seepage flow along its negative direction when a second-order tensor is used to express the TPG. Although there are amount of debates about the nature of the TPG, it is no doubt that this obstruction changes with the direction of the seepage velocity. Otherwise, there will be problems like the vector representation. As a TPG in different directions act as a vector, according to the tensor identification theorem (Huang et al., 2003), it is a second-order tensor. It should be noted from Eq. (8) that when a TPG is represented by a second-order tensor proposed in this paper, it always acts as a barrier to seepage flow. Therefore, the assumption in this article is valid. However, this hypothesis is not the final solution. Of course, other assumptions can also be made. For example, it can be assumed that the effective pressure gradient under a TPG is:

$$
\boldsymbol{J}_{e}=\boldsymbol{J}-\boldsymbol{\lambda} \cdot \frac{\boldsymbol{K} \cdot \boldsymbol{J}}{|\boldsymbol{K} \cdot \boldsymbol{J}|}
$$

In fact, this assumption is also taken in the derivation of some preceding cases, but it is required to be true simultaneously with the hypothesis Eq. (6). Only using the hypothesis Eq. (41), a SGE can also be obtained, and the corresponding seepage initiation condition is written as follows:

$$
\left\{\begin{array}{l}
\sqrt{\left(K_{x} \frac{\partial p}{\partial x}\right)^{2}+\left(K_{y} \frac{\partial p}{\partial y}\right)^{2}+\left(K_{z} \frac{\partial p}{\partial z}\right)^{2}}>\lambda_{x} K_{x} \\
\sqrt{\left(K_{x} \frac{\partial p}{\partial x}\right)^{2}+\left(K_{y} \frac{\partial p}{\partial y}\right)^{2}+\left(K_{z} \frac{\partial p}{\partial z}\right)^{2}}>\lambda_{y} K_{y} \\
\sqrt{\left(K_{x} \frac{\partial p}{\partial x}\right)^{2}+\left(K_{y} \frac{\partial p}{\partial y}\right)^{2}+\left(K_{z} \frac{\partial p}{\partial z}\right)^{2}}>\lambda_{z} K_{z}
\end{array}\right.
$$

It can also be assumed that the effective pressure gradient under a TPG tensor is:

$$
\boldsymbol{J}_{e}=\boldsymbol{J}-\boldsymbol{\lambda} \cdot \frac{\boldsymbol{J}}{|\boldsymbol{J}|}
$$

The corresponding seepage initiation condition is:

$$
\left\{\begin{array}{l}
\sqrt{\left(\frac{\partial p}{\partial x}\right)^{2}+\left(\frac{\partial p}{\partial y}\right)^{2}+\left(\frac{\partial p}{\partial z}\right)^{2}}>\lambda_{x} \\
\sqrt{\left(\frac{\partial p}{\partial x}\right)^{2}+\left(\frac{\partial p}{\partial y}\right)^{2}+\left(\frac{\partial p}{\partial z}\right)^{2}}>\lambda_{y} \\
\sqrt{\left(\frac{\partial p}{\partial x}\right)^{2}+\left(\frac{\partial p}{\partial y}\right)^{2}+\left(\frac{\partial p}{\partial z}\right)^{2}}>\lambda_{z}
\end{array}\right.
$$

It can be verified that both assumptions (41) and (43) ensure that the TPG always acts as a barrier to seepage flow and that both can be degenerated into isotropic reservoir cases. As for which assumption is more reasonable, it needs further investigations. We hope this article can play an essential role in attracting valuable opinions.

\subsection{Prospect}

The TPG for a single-phase fluid flow in porous media is controversial. This controversy mainly focused on the flow of low-viscosity crude oil in low permeability reservoirs. High viscous heavy oils containing amount of bitumen and tar are generally considered to be Bingham fluids, and their constitutive equations can be appropriately simplified to be consistent with the constitutive equations with a TPG mentioned herein (Wang et al., 2013). Therefore, the equations presented in this study can be used in this case (Liu, 2013).

It has already been mentioned that the 2-dimensional or 3dimensional SGEs with a TPG are generally very complicated. We propose to solve the equation sets with pressure and seepage velocity instead of the SGEs. Due to the existence of seepage initiation conditions, this is a moving boundary problem. Their numerical solution is not easy. Currently, the numerical methods are mainly used to solve its 1-dimensional problem. For 2-dimensional or 3-dimensional problems, the numerical solution method will be much difficult and requires further study.

The current measurement of a TPG assumes that the reservoir is isotropic (Ding et al., 2014; Tian et al., 2018). When reservoirs are anisotropic, this actually tests the projection of the TPG in one direction. For anisotropic reservoirs, measurement of its TPG has not been reported. If it is possible to determine its three principal axes from geology, core analysis, or geophysics tests, the difficulty of tests will be reduced. It can found from Eq. (11) that if the pressure gradient is along a principal axis, the seepage velocity expression in this direction is the same as that for isotropic reservoirs. Therefore, a feasible scheme is to sample in the direction of the three principal axes and test the TPG in the same way as isotropic reservoirs in each direction. When the directions of the principal axes are 
impossible to be determined, since a TPG has 9 components, it is necessary to sample at least in 9 directions. A viable scheme is to sample at $60^{\circ}$ intervals in each coordinate plane and ensure that the 9 directions are completely different. If only one principal axis can be determined, the scheme is to sample along the principal axis and take 4 samples at $45^{\circ}$ intervals in the plane perpendicular to the principal axis. Each sample is tested according to the method for isotropic reservoir samples. Using the TPGs in each direction, the TPG tensor can be obtained according to the coordinate transformation law of a tensor. It can be concluded that determining permeability principal axes is the key to determining the TPG of anisotropic reservoirs. Otherwise, it will be very difficult. The method proposed here is only an idea, and it requires future practical work.

\section{Conclusions}

In this paper, the reservoir SGEs with a TPG are studied, and the SGEs for isotropic and anisotropic low-permeability reservoirs are obtained in Cartesian, cylindrical coordinate systems. Then, the results are discussed extensively, and the following conclusions are obtained:

1) A TPG can not be represented by a vector, but a secondorder tensor. The form of the effective pressure gradient for anisotropic reservoirs requires further investigation.

2) The equations of isotropic low-permeability reservoirs in Cartesian coordinate systems derived here are consistent with these in cylindrical coordinate systems, and the inconsistency in the literature between the two is solved.

3) The SEGs for anisotropic reservoirs with a TPG derived here can be degenerated into isotropic conditions.

4) The condition of seepage initiation for anisotropic reservoirs with a TPG is derived under the general case. According to different assumptions, specific initiation conditions will be obtained from it.

5) The SGE for low-permeability with a TPG is very complicated in Cartesian and cylindrical coordinate systems. Only in the case of radial flow, a simple form of a SGE with a TPG in cylindrical coordinate systems is obtained.

6) In general, the SGEs with a TPG are very complex and are not suitable for numerical simulation analysis. It is recommended to use the equation set with the seepage velocity and pressure as the variables for simulations.

\section{Nomenclature}

\section{Latin}

$p=$ pore pressure, $\mathrm{Pa}$

$\boldsymbol{K}, K=$ permeability tensor and permeability scalar (for 1-D or isotropic), respectively, $\mathrm{m}^{2}$

$K_{i j}=$ component of the permeability tensor, subscript $i$ and $j$ are $x, y$ or $z, \mathrm{~m}^{2}$

$\boldsymbol{V}, V=$ seepage velocity vector and seepage velocity (for 1-D), respectively, $\mathrm{m} / \mathrm{s}$

$J, J=$ pressure gradient vector and pressure gradient (for 1-D), respectively, $\mathrm{Pa} / \mathrm{m}$

$J_{e}=$ effective pressure gradient vector, $\mathrm{Pa} / \mathrm{m}$
$V_{x}, V_{y}=$ components of seepage velocity in $x, y$ directions, respectively, $\mathrm{m} / \mathrm{s}$

$V_{r}, V_{\theta}=$ components of seepage velocity in $r, \theta$ directions, respectively, $\mathrm{m} / \mathrm{s}$

$V_{z}=$ component of seepage velocity in $z$ direction in cylindrical or Cartesian coordinate systems, $\mathrm{m} / \mathrm{s}$

$c_{t}=$ total compressibility, $1 / \mathrm{Pa}$

$c_{r}=$ pore compressibility, $1 / \mathrm{Pa}$

$c_{f}=$ fluid compressibility, $1 / \mathrm{Pa}$

$q=$ source or sink item, $\mathrm{m}^{3} /\left(\mathrm{m}^{3} \cdot \mathrm{s}\right)$

$x, y=$ coordinates of Cartesian coordinate systems, $\mathrm{m}$

$r=$ coordinate of cylindrical coordinate systems, $\mathrm{m}$

$z=$ coordinate of cylindrical or Cartesian coordinate systems, $\mathrm{m}$

$e_{i}, \bar{e}_{i}=$ unit basis vector in cylindrical coordinate systems and Cartesian coordinate systems, respectively, subscript $i$ denotes $r, \theta, z$ or $x, y, z$

\section{Greek}

$\lambda, \lambda=$ TPG tensor and TPG scalar (for 1-D or isotropic), respectively, $\mathrm{Pa} / \mathrm{m}$

$\lambda_{x}, \lambda_{y}, \lambda_{z}=$ components of TPG tensors in $x, y, z$-direction, respectively, $\mathrm{Pa} / \mathrm{m}$

$\phi=$ porosity, $\%$

$\mu=$ fluid viscosity, $\mathrm{Pa} \cdot \mathrm{s}$

$\rho=$ fluid density, $\mathrm{kg} / \mathrm{m}^{3}$

$\theta=$ coordinates of cylindrical coordinate systems

\section{Acknowledgments}

The first author acknowledges the National Natural Science Foundation of China (Grant No.11602276) for the financial support to this research.

Open Access This article is distributed under the terms and conditions of the Creative Commons Attribution (CC BY-NC-ND) license, which permits unrestricted use, distribution, and reproduction in any medium, provided the original work is properly cited.

\section{References}

Chen, Y. Improper use of the starting pressure gradient of linear flow in the plane radial flow equation. Acta Petrolei Sinica 2011, 32(6): 1088-1092. (in Chinese)

Civan, F. Modeling gas flow through hydraulically-fractured shale-gas reservoirs involving molecular-to-inertial transport regimes and threshold-pressure gradient. Paper SPE 166324 Presented at SPE Annual Technical Conference and Exhibition, New Orleans, Louisiana, USA, 30 September-2 October, 2013.

Civan, F. Effect of poroelasticity, pore confinement, molecularto-inertial transport, and threshold pressure on flow of gas through hydraulically-fractured shale-gas reservoirs. Paper SPE 187056 Presented at SPE Annual Technical Conference and Exhibition, San Antonio, Texas, USA, 9-11 October, 2017.

Ding, J., Yang, S., Nie, X., et al. Dynamic threshold pressure gradient in tight gas reservoir. J. Nat. Gas Sci. Eng. 2014, 20(2): 155-160.

Diwu, P., Liu, T., You, Z., et al. Effect of low velocity non- 
Darcy flow on pressure response in shale and tight oil reservoirs. Fuel 2018, 216: 398-406.

Dou, H., Ma, S., Zou, C., et al. Threshold pressure gradient of fluid flow through multi-porous media in low and extralow permeability reservoirs. Sci. China Earth Sci. 2014, 57(11): 2808-2818.

Dudgeon, C.R. An experimental study of the flow of water through coarse granular media. La Houille Blanche 2010, 21(7): 785-801.

Escobar, F.H., Zhao, Y.L., Pournik, M., et al. Interpretation of pressure tests in uniform-flux fractured vertical wells with threshold pressure gradient in low permeability reservoirs. ARPN J. Eng. Appl. Sci. 2015, 10(20): 93649372.

Guo, J., Zhang, S., Zhang, L., et al. Well testing analysis for horizontal well with consideration of threshold pressure gradient in tight gas reservoirs. J. Hydrodyn. 2012, 24(4): 561-568.

Huang, K., Xue, M., Lu, M. Tensor Analysis. Beijing, Tsinghua University Press, 2003. (in Chinese)

Kong, X. Advanced Mechanics of Flow in Porous Media (2ed). Hefei, Press of University of Science and Technology of China, 2010. (in Chinese)

Li, D., Zha, W., Liu, S., et al. Pressure transient analysis of low permeability reservoir with pseudo threshold pressure gradient. J. Pet. Sci. Eng. 2016, 147: 308-316.

Li, S., Cheng, L., Li, X., et al. Nonlinear seepage flow of ultralow permeability reservoirs. Pet. Explor. Dev. 2008, 35(5): 606-612.

Liu, H. The numerical simulation for multistage fractured horizontal well in low-permeability reservoirs based on modified Darcy's equation. J. Pet. Explor. Prod. Technol. 2017, 7(3): 735-746.

Liu, H., Wu, S. The numerical simulation for multi-stage fractured horizontal well in low permeability reservoirs based on modified Darcy's equation. Paper SPE 176269 Presented at SPE/IATMI Asia Pacific Oil \& Gas Conference and Exhibition, Nusa Dua, Bali, Indonesia, 20-22 October, 2015.

Liu, W. Numerical simulation of nonlinear seepage flow in low-permeable reservoirs with moving boundaries. Qingdao, China University of Petroleum Doctoral Dissertation, 2013. (in Chinese)

Liu, W., Yao, J., Chen, Z., et al. Effect of quadratic pressure gradient term on a one-dimensional moving boundary problem based on modified Darcy's law. Acta Mech. Sin. 2016, 32(1): 38-53.

Liu, W., Yao, J., Wang, Y. Exact analytical solutions of moving boundary problems of one-dimensional flow in semi-infinite long porous media with threshold pressure gradient. Int. J. Heat Mass Transf. 2012, 55(21-22): 60176022.

Lu, J. Pressure behavior of a hydraulically fractured well in tight gas formation with threshold pressure gradient. Paper SPE 152158 Presented at SPE Middle East Unconventional Gas Conference and Exhibition, Abu Dhabi, UAE, 23-25 January, 2012.
Lu, J. Pressure behavior of fractured gas wells in lowpermeability reservoirs with threshold pressure gradient. STRPM 2014, 5(2): 171-178.

Prada, A., Civan, F. Modification of Darcy's law for the threshold pressure gradient. J. Pet. Sci. Eng. 1999, 22(4): 237-240.

Song, H., Cao, Y., Yu, M., et al. Impact of permeability heterogeneity on production characteristics in waterbearing tight gas reservoirs with threshold pressure gradient. J. Nat. Gas Sci. Eng. 2015, 22: 172-181.

Sun, Q., Zhang, R., Zhang, Y., et al. Pressure transient analysis of the low permeability composite reservoir with threshold pressure gradient. Presented at Asia-Pacific Power and Energy Engineering Conference, Chengdu, China, 28-31 March, 2010.

Thomas, L.K., Katz, D.L., Tek, M.R. Threshold pressure phenomena in porous media. Soc. Petrol. Eng. J. 1968, 8(2): 174-184.

Tian, W., Li, A., Ren, X., et al. The threshold pressure gradient effect in the tight sandstone gas reservoirs with high water saturation. Fuel 2018, 226: 221-229.

Wang, X., Hao, M., Han, Y. Implication of the threshold pressure gradient and its application. Acta Petrolei Sinica 2013, 34(1): 188-191. (in Chinese)

Wang, X., Sheng, J. Effect of low-velocity non-darcy flow on well production performance in Shale and Tight oil reservoirs. Fuel 2017, 190: 41-46.

Wang, X., Zhu, G., Wang, L. Exact analytical solutions for moving boundary problems of one-dimensional flow in semi-infinite porous media with consideration of threshold pressure gradient. J. Hydrodyn. Ser. B 2015, 27(4): 542-547.

Wu, K., Li, X., Yang, P., et al. The Establishment of a novel deliverability equation of abnormal pressure gas reservoirs considering a variable threshold pressure drop. Pet. Sci. Technol. 2014, 32(1): 15-21.

Xiong, W., Lei, Q., Gao, S., et al. Pseudo threshold pressure gradient to flow for low permeability reservoirs. Pet. Explor. Dev. 2009, 36(2): 232-236.

$\mathrm{Xu}$, J., Jiang, R., Xie, L., et al. Non-Darcy flow numerical simulation for low-permeability reservoirs. Paper SPE 154890 Presented at SPE Europec/EAGE Annual Conference, Copenhagen, Denmark, 4-7 June, 2012.

Zeng, B., Cheng, L., Hao, F. Experiment and mechanism analysis on threshold pressure gradient with different fluids. Paper SPE 140678 Presented at Nigeria Annual International Conference and Exhibition, Tinapa-Calabar, Nigeria, 31 July-7 August, 2010.

Zeng, B., Cheng, L., Li, C. Low velocity non-linear flow in ultra-low permeability reservoir. J. Pet. Sci. Eng. 2011, 80(1): 1-6.

Zeng, J., Wang, X., Guo, J., et al. Analytical model for multifractured horizontal wells in tight sand reservoir with threshold pressure gradient. Paper SPE 181819 Presented at SPE Asia Pacific Hydraulic Fracturing Conference, Beijing, China, 24-26 August, 2016.

Zeng, J., Wang, X., Guo, J., et al. Composite linear flow model for multi-fractured horizontal wells in tight sand 
reservoirs with the threshold pressure gradient. J. Pet. Sci. Eng. 2018, 165: 890-912.

Zhao, Y., Escobar, F.H., Jamili, A., et al. Effect of wellbore storage on the vertical well pressure behavior with threshold pressure gradient in low permeability reservoirs. ARPN-JEAS 2015a, 10(22): 10659-10665.

Zhao, Y., Zhang, L., Wu, F., et al. Analysis of horizontal well pressure behaviour in fractured low permeability reservoirs with consideration of the threshold pressure gradient. J. Geophys. Eng. 2013, 10(3): 35014-35023.

Zhao, Y., Zhang, L., Zhao, J., et al. Transient pressure analysis of horizontal well in low permeability oil reservoir. Int. J. Oil Gas Coal Technol. 2015b, 10(1): 23-38.

Zhu, W., Song, H., Huang, X., et al. Pressure characteristics and effective deployment in a water-bearing tight gas reservoir with low-velocity non-darcy flow. Energy Fuels 2011, 25(3): 1111-1117. 
Appendix 1: Governing equations with a TPG for isotropic reservoirs in a Cylindrical coordinate system

According to Eq. (2), in the cylindrical coordinate system $(r, \theta, z)$, the seepage velocity has a general form of:

$$
\left\{\begin{array}{l}
V_{r}=-\frac{K}{\mu} \frac{\partial p}{\partial r}\left(1-\frac{\lambda}{\sqrt{\left(\frac{\partial p}{\partial r}\right)^{2}+\frac{1}{r^{2}}\left(\frac{\partial p}{\partial \theta}\right)^{2}+\left(\frac{\partial p}{\partial z}\right)^{2}}}\right) \\
V_{\theta}=-\frac{K}{\mu} \frac{\partial p}{r \partial \theta}\left(1-\frac{\lambda}{\sqrt{\left(\frac{\partial p}{\partial r}\right)^{2}+\frac{1}{r^{2}}\left(\frac{\partial p}{\partial \theta}\right)^{2}+\left(\frac{\partial p}{\partial z}\right)^{2}}}\right), \quad \sqrt{\left(\frac{\partial p}{\partial r}\right)^{2}+\frac{1}{r^{2}}\left(\frac{\partial p}{\partial \theta}\right)^{2}+\left(\frac{\partial p}{\partial z}\right)^{2}}>\lambda \\
V_{z}=-\frac{K}{\mu} \frac{\partial p}{\partial z}\left(1-\frac{\lambda}{\sqrt{\left(\frac{\partial p}{\partial r}\right)^{2}+\frac{1}{r^{2}}\left(\frac{\partial p}{\partial \theta}\right)^{2}+\left(\frac{\partial p}{\partial z}\right)^{2}}}\right)
\end{array}\right.
$$

The general form of the SGE in the cylindrical coordinate system can also be derived similar to the derivation of that in the Cartesian coordinate system. For simplicity, the SGE is derived for radial flow without any source or sinks term, considering that the radial SGE is widely used in well test analysis. Thus, the continuity Eq. (16) can be written as:

$$
\frac{\partial(\phi \rho)}{\partial t}+\frac{1}{r} \frac{\partial}{\partial r}\left(\rho r V_{r}\right)=0
$$

Only considering the radial velocity, Eq. (A1-1) can be simplified as:

$$
V_{r}=-\frac{K}{\mu} \frac{\partial p}{\partial r}\left(1-\frac{\lambda}{\left|\frac{\partial p}{\partial r}\right|}\right)
$$

Substituting Eq. (A1-3) into continuity Eq. (A1-2), it yields:

$$
\frac{1}{r} \frac{\partial}{\partial r}\left(\rho r V_{r}\right)=-\frac{K}{\mu} \frac{\rho}{r}\left\{\left[\frac{\partial p}{\partial r}+c_{f} r\left(\frac{\partial p}{\partial r}\right)^{2}\right]\left(1-\frac{\lambda}{\left|\frac{\partial p}{\partial r}\right|}\right)+r \frac{\partial^{2} p}{\partial r^{2}}\right\}
$$

Ignoring the quadratic term of the pressure gradient similar to general treatments, it yields:

$$
\frac{1}{r} \frac{\partial}{\partial r}\left(\rho r V_{r}\right)=-\frac{\rho K}{\mu}\left(\frac{1}{r} \frac{\partial p}{\partial r}-\frac{\lambda}{r} \frac{\partial p}{\partial r} \frac{1}{\left|\frac{\partial p}{\partial r}\right|}+\frac{\partial^{2} p}{\partial r^{2}}\right)
$$

Therefore, the SGE for radial flow with a TPG in the cylindrical coordinate system can be written as:

$$
\frac{\partial^{2} p}{\partial r^{2}}+\frac{1}{r} \frac{\partial p}{\partial r}-\frac{\lambda}{r} \frac{\partial p}{\partial r} \frac{1}{\left|\frac{\partial p}{\partial r}\right|}=\frac{\phi \mu c_{t}}{K} \frac{\partial p}{\partial t}, \quad\left|\frac{\partial p}{\partial r}\right|>\lambda
$$

\section{Appendix 2: Governing equations with a TPG for anisotropic reservoirs}

\section{Appendix 2.1: Cartesian coordinate system}

Using Eqs. (21) and (7), the seepage velocity of anisotropic reservoir with a TPG in the Cartesian coordinate system can be written as:

$$
\mathbf{V}=-\frac{1}{\mu}\left\{\left(\begin{array}{c}
K_{x} \frac{\partial p}{\partial x} \\
K_{y} \frac{\partial p}{\partial y} \\
K_{z} \frac{\partial p}{\partial z}
\end{array}\right)-\left[\begin{array}{l}
\lambda_{x} K_{x}^{2} \frac{\partial p}{\partial x} \\
\lambda_{y} K_{y}^{2} \frac{\partial p}{\partial y} \\
\lambda_{z} K_{z}^{2} \frac{\partial p}{\partial z}
\end{array}\right] \frac{1}{\sqrt{\left(K_{x} \frac{\partial p}{\partial x}\right)^{2}+\left(K_{y} \frac{\partial p}{\partial y}\right)^{2}+\left(K_{z} \frac{\partial p}{\partial z}\right)^{2}}}\right\}
$$


It also can be written as follows:

$$
\left[\begin{array}{l}
V_{x} \\
V_{y} \\
V_{z}
\end{array}\right]=-\frac{1}{\mu}\left[\begin{array}{l}
\left(1-\frac{\lambda_{x} K_{x}}{\sqrt{\left(K_{x} \frac{\partial p}{\partial x}\right)^{2}+\left(K_{y} \frac{\partial p}{\partial y}\right)^{2}+\left(K_{z} \frac{\partial p}{\partial z}\right)^{2}}}\right) K_{x} \frac{\partial p}{\partial x} \\
\left.1-\frac{\lambda_{y} K_{y}}{\sqrt{\left(K_{x} \frac{\partial p}{\partial x}\right)^{2}+\left(K_{y} \frac{\partial p}{\partial y}\right)^{2}+\left(K_{z} \frac{\partial p}{\partial z}\right)^{2}}}\right) K_{y} \frac{\partial p}{\partial y} \\
\left(1-\frac{\lambda_{z} K_{z}}{\sqrt{\left(K_{x} \frac{\partial p}{\partial x}\right)^{2}+\left(K_{y} \frac{\partial p}{\partial y}\right)^{2}+\left(K_{z} \frac{\partial p}{\partial z}\right)^{2}}}\right) K_{z} \frac{\partial p}{\partial z}
\end{array}\right]
$$

If Eqs. (21) and (A2.1-2) holds, then the relational expression $K_{x} \lambda_{x}=K_{y} \lambda_{y}=K_{z} \lambda_{z}$ holds. Eq. (A2.1-2) can also be written in another form as:

$$
\left\{\begin{array}{l}
V_{x}=-\frac{K_{x}}{\mu}\left(\frac{\partial p}{\partial x}-\lambda_{\lambda x}\right) \\
V_{y}=-\frac{K_{y}}{\mu}\left(\frac{\partial p}{\partial z}-\lambda_{\lambda y}\right), \quad \sqrt{\left(K_{x} \frac{\partial p}{\partial x}\right)^{2}+\left(K_{y} \frac{\partial p}{\partial y}\right)^{2}+\left(K_{z} \frac{\partial p}{\partial z}\right)^{2}}>K_{x} \lambda_{x}=K_{y} \lambda_{y}=K_{z} \lambda_{z} \\
V_{z}=-\frac{K_{z}}{\mu}\left(\frac{\partial p}{\partial z}-\lambda_{\lambda z}\right)
\end{array}\right.
$$

For the continuity Eq. (16) in the $x$ direction, the following holds:

$$
\frac{\partial\left(\rho V_{x}\right)}{\partial x}=-\frac{K_{x}}{\mu} \frac{\partial}{\partial x}\left(\rho \frac{\partial p}{\partial x}-\rho \lambda_{\lambda x}\right)=-\frac{K_{x} \rho}{\mu}\left[c_{f}\left(\frac{\partial p}{\partial x}\right)^{2}+\frac{\partial^{2} p}{\partial x^{2}}-\frac{\partial \lambda_{\lambda x}}{\partial x}-c_{f} \lambda_{\lambda x} \frac{\partial p}{\partial x}\right]
$$

Ignoring the quadratic term of the pressure gradient, and then substituting Eq. (12) into Eq. (A2.1-4), it yields:

$$
\frac{\partial\left(\rho V_{x}\right)}{\partial x}=-\frac{\rho K_{x}}{\mu}\left\{\begin{array}{l}
\frac{\partial^{2} p}{\partial x^{2}}\left(1-\frac{\lambda_{x} K_{x}}{\sqrt{\left(K_{x} \frac{\partial p}{\partial x}\right)^{2}+\left(K_{y} \frac{\partial p}{\partial y}\right)^{2}+\left(K_{z} \frac{\partial p}{\partial z}\right)^{2}}}\right) \\
+\frac{\lambda_{x} K_{x}}{\partial x} \frac{\left[\left(K_{x} \frac{\partial p}{\partial x}\right)^{2}+\left(K_{y} \frac{\partial p}{\partial y}\right)^{2}+\left(K_{z} \frac{\partial p}{\partial z}\right)^{2}\right]^{\frac{3}{2}} \times}{\left[K_{x}^{2} \frac{\partial p}{\partial x} \frac{\partial^{2} p}{\partial x^{2}}+K_{y}^{2} \frac{\partial p}{\partial y} \frac{\partial^{2} p}{\partial x \partial y}+K_{z}^{2} \frac{\partial p}{\partial z} \frac{\partial^{2} p}{\partial x \partial z}\right)}
\end{array}\right\}
$$

Similar results can be obtained in $y$ and $z$ directions, which are not explicitly written here. Assuming that the source or sink term is 0 and second order continuous derivatives with respect to $x, y$ and $z$ exist, the governing equation can be written 
as:

$$
\begin{aligned}
& \left(\frac{\partial^{2} p}{\partial x^{2}}+\frac{\partial^{2} p}{\partial y^{2}}+\frac{\partial^{2} p}{\partial z^{2}}\right)\left(1-\frac{\lambda_{x} K_{x}}{\sqrt{\left(K_{x} \frac{\partial p}{\partial x}\right)^{2}+\left(K_{y} \frac{\partial p}{\partial y}\right)^{2}+\left(K_{z} \frac{\partial p}{\partial z}\right)^{2}}}\right) \\
& +\frac{\lambda_{x} K_{x}}{\left[\left(K_{x} \frac{\partial p}{\partial x}\right)^{2}+\left(K_{y} \frac{\partial p}{\partial y}\right)^{2}+\left(K_{z} \frac{\partial p}{\partial z}\right)^{2}\right]^{\frac{3}{2}}}\left[\begin{array}{c}
K_{x}^{2}\left(\frac{\partial p}{\partial x}\right)^{2} \frac{\partial^{2} p}{\partial x^{2}} \\
+K_{y}^{2}\left(\frac{\partial p}{\partial y}\right)^{2} \frac{\partial^{2} p}{\partial y^{2}} \\
+K_{z}^{2}\left(\frac{\partial p}{\partial z}\right)^{2} \frac{\partial^{2} p}{\partial z^{2}} \\
+\left(K_{x}^{2}+K_{y}^{2}\right) \frac{\partial p}{\partial x} \frac{\partial p}{\partial y} \frac{\partial^{2} p}{\partial x \partial y} \\
+\left(K_{x}^{2}+K_{z}^{2}\right) \frac{\partial p}{\partial x} \frac{\partial p}{\partial z} \frac{\partial^{2} p}{\partial x \partial z} \\
+\left(K_{y}^{2}+K_{z}^{2}\right) \frac{\partial p}{\partial y} \frac{\partial p}{\partial z} \frac{\partial^{2} p}{\partial y \partial z}
\end{array}\right]=\frac{\phi \mu c_{t}}{K t} \frac{\partial p}{\partial t} \\
& \sqrt{\left(K_{x} \frac{\partial p}{\partial x}\right)^{2}+\left(K_{y} \frac{\partial p}{\partial y}\right)^{2}+\left(K_{z} \frac{\partial p}{\partial z}\right)^{2}}>K_{x} \lambda_{x}=K_{y} \lambda_{y}=K_{z} \lambda_{z}
\end{aligned}
$$

Eq. (A2.1-6) is a SGE for permeability anisotropic reservoirs with a TPG and its initiation condition in the Cartesian coordinate system when $K_{x} \lambda_{x}=K_{y} \lambda_{y}=K_{z} \lambda_{z}$.

\section{Appendix 2.2: Cylindrical coordinate system}

When the flow is radial, the seepage velocity vector in cylindrical coordinate systems could be expressed as:

$$
\boldsymbol{V}=\left[\begin{array}{c}
V_{r} \\
V_{\theta} \\
V_{z}
\end{array}\right]=\left[\begin{array}{c}
V_{r} \\
0 \\
0
\end{array}\right]
$$

The pressure gradient in the cylindrical coordinate system is:

$$
\boldsymbol{J}=\left[\begin{array}{c}
\frac{\partial p}{\partial r} \\
\frac{1}{r} \frac{\partial p}{\partial \theta} \\
\frac{\partial p}{\partial z}
\end{array}\right]
$$

Therefore, the 3-dimensional domain seepage velocity vector is:

$$
\begin{aligned}
& \boldsymbol{V}=-\frac{\boldsymbol{K}}{\mu} \cdot\left(\boldsymbol{J}+\boldsymbol{\lambda} \cdot \frac{\boldsymbol{V}}{|\boldsymbol{V}|}\right)= \\
& -\frac{1}{\mu}\left[\begin{array}{c}
\frac{\partial p}{\partial r}\left(K_{x} \cos ^{2} \theta+K_{y} \sin ^{2} \theta\right)+\frac{1}{r} \frac{\partial p}{\partial \theta}\left(K_{y}-K_{x}\right) \cos \theta \sin \theta+\frac{V_{r}}{\left|V_{r}\right|}\left(K_{y} \lambda_{y} \sin ^{2} \theta+K_{x} \lambda_{x} \cos ^{2} \theta\right) \\
\frac{\partial p}{\partial r}\left(K_{y}-K_{x}\right) \cos \theta \sin \theta+\frac{1}{r} \frac{\partial p}{\partial \theta}\left(K_{x} \sin ^{2} \theta+K_{y} \cos ^{2} \theta\right)+\frac{V_{r}}{\left|V_{r}\right|}\left(K_{y} \lambda_{y}-K_{x} \lambda_{x}\right) \cos \theta \sin \theta \\
K_{z} \frac{\partial p}{\partial z}
\end{array}\right]
\end{aligned}
$$


The same assumptions are made as in the case of derivation in Cartesian coordinate system that the direction of seepage velocity with a TPG is the same as that of without a TPG, i.e., $K_{y} \lambda_{y}=K_{x} \lambda_{x}$. Therefore, Eq. (A2.2-3) can be rewritten as:

$$
\left[\begin{array}{c}
V_{r} \\
0 \\
0
\end{array}\right]=-\frac{1}{\mu}\left[\begin{array}{c}
\frac{\partial p}{\partial r}\left(K_{x} \cos ^{2} \theta+K_{y} \sin ^{2} \theta\right)+\frac{1}{r} \frac{\partial p}{\partial \theta}\left(K_{y}-K_{x}\right) \cos \theta \sin \theta+\frac{V_{r}}{\left|V_{r}\right|} K_{y} \lambda_{y} \\
\frac{\partial p}{\partial r}\left(K_{y}-K_{x}\right) \cos \theta \sin \theta+\frac{1}{r} \frac{\partial p}{\partial \theta}\left(K_{x} \sin ^{2} \theta+K_{y} \cos ^{2} \theta\right) \\
K_{z} \frac{\partial p}{\partial z}
\end{array}\right]
$$

From Eq. (A2.2-4), the seepage velocity $\left(\boldsymbol{V}_{r}\right)$ can be written as:

$$
\left\{\begin{array}{l}
V_{r}=-\frac{1}{\mu} \frac{\partial p}{\partial r}\left(\frac{K_{x} K_{y}}{K_{x} \sin ^{2} \theta+K_{y} \cos ^{2} \theta}-\frac{K_{y} \lambda_{y}}{\left|\frac{\partial p}{\partial r}\right|}\right) \\
\frac{\partial p}{\partial z}=0, \\
\frac{1}{r} \frac{\partial p}{\partial \theta}=-\frac{\partial p}{\partial r} \frac{\partial}{\partial r} \frac{\lambda_{y}}{K_{x}}\left(K_{x} \sin ^{2} \theta+K_{x}\right) \cos \theta \sin \theta \\
\sin ^{2} \theta+K_{y} \cos ^{2} \theta
\end{array}\right.
$$

Substituting Eq. (A2.2-5) into the continuity Eq. (A1-2) for radial flow without considering the source or sink term $q$ and ignoring the quadratic term of the pressure gradient, it yields:

$$
\left\{\begin{array}{l}
\frac{\partial^{2} p}{\partial r^{2}} \frac{K_{x} K_{y}}{K_{x} \sin ^{2} \theta+K_{y} \cos ^{2} \theta}+\frac{1}{r} \frac{\partial p}{\partial r}\left(\frac{K_{x} K_{y}}{K_{x} \sin ^{2} \theta+K_{y} \cos ^{2} \theta}-\frac{K_{y} \lambda_{y}}{\left|\frac{\partial p}{\partial r}\right|}\right)=\phi \mu c_{t} \frac{\partial p}{\partial t} \\
\left|\frac{\partial p}{\partial r}\right|>\frac{\lambda_{y}}{K_{x}}\left(K_{x} \sin ^{2} \theta+K_{y} \cos ^{2} \theta\right)
\end{array}\right.
$$

Eq. (A2.2-6) is the SGE with a TPG for the radial flow of anisotropic reservoirs when $K_{x} \lambda_{x}=K_{y} \lambda_{y}$. 